\title{
Occurrence and Transport of Perfluoroalkyl Acids (PFAAs), Including Short-Chain PFAAs in Tangxun Lake, China
}

\author{
Zhen Zhou, ${ }^{\dagger}$ Yong Liang, ${ }^{\ddagger} \S$ Yali Shi, ${ }^{*}{ }^{\dagger}$ Lin $\mathrm{Xu}^{\dagger}{ }^{\dagger}$ and Yaqi Cai ${ }^{\dagger}$ \\ ${ }^{\dagger}$ State Key Laboratory of Environmental Chemistry and Ecotoxicology, Research Center for Eco-Environmental Science, Chinese \\ Academy of Sciences, Beijing 100085, China \\ ${ }^{\ddagger}$ School of Medicine, Jianghan University, Hubei Province, Wuhan 430056, China \\ ${ }^{\S}$ Key Laboratory of Optoelectronic Chemical Materials and Devices of Ministry of Education, Jianghan University, Hubei Province, \\ Wuhan 430056, China
}

\section{Supporting Information}

ABSTRACT: Short-chain perfluoroalkyl acids (PFAAs), which have less than seven fluorinated carbons, have been introduced as substitutes for eight-carbon homologue products. In this study, water, sediment, and biological samples (fish and plant) were collected from Tangxun Lake, which is located near a production base of the fluorochemical industry in Wuhan, China. Perfluorobutane sulfonate (PFBS) and perfluorobutanoic acid (PFBA) were the predominant PFAAs in surface water, with average concentrations of $3660 \mathrm{ng} / \mathrm{L}$ and $4770 \mathrm{ng} / \mathrm{L}$, respectively. However, perfluorooctane sulfonate (PFOS) was the most abundant PFAA in sediments, with an average concentration of $74.4 \mathrm{ng} / \mathrm{g} \mathrm{dw}$. The organic carbon normalized distribution coefficients $\left(K_{\mathrm{OC}}\right)$ indicated that shortchain PFAAs $\left(\mathrm{CF}_{2}<7\right)$ tended to have lower adsorption potentials than PFOS, perfluorooctanoic acid (PFOA), and longer perfluoroalkyl chain

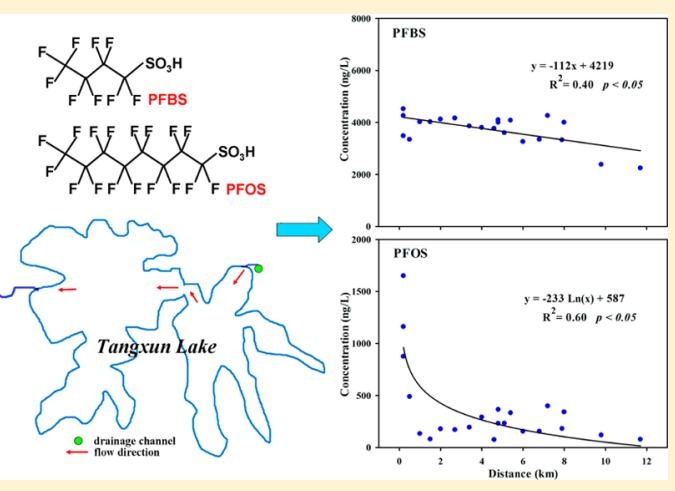
compounds. PFBS and PFBA could transport to a farther distance in the horizontal direction along the water flow and infiltrate into deeper depths in the vertical direction. However, levels of PFOS and PFOA in water dropped exponentially along the current, and their proportions were decreased gradually with the increasing depth in sediment cores. Furthermore, values of log bioconcentration factor $(\mathrm{BCF})$ of the short-chain PFAAs were all relatively low $(<1)$, indicating no bioaccumulation potentials for short-chain PFAAs in aquatic species.

\section{INTRODUCTION}

Perfluoroalkyl acids (PFAAs) have been widely used in industrial and household products, including surfactants, firefighting foams, coatings, and pesticides, due to their extreme thermal and chemical stability and hydro- and lipophobic properties. ${ }^{1,2}$ However, PFAAs have been characterized as global environmental contaminants and are frequently detected in land, oceans, polar regions, wildlife, and humans. ${ }^{3-7}$ At the fourth meeting of the Parties of the Stockholm Convention, perfluorooctane sulfonate (PFOS), its salts, and perfluorooctane sulfonyl fluoride (PFOSF) were listed as new persistent organic pollutants (POPs). ${ }^{8}$ As the major global producer, $3 \mathrm{M}$ Company ceased production of PFOSF in 2002. However, several other companies worldwide continue to produce PFAAs, and especially in China their production has rapidly increased. In 2008, the annual production of PFOSF in China was about 250 tons, approximately half of which was used domestically. ${ }^{9}$ Meanwhile, short-chain PFAAs, which have less than seven fluorinated carbons, have been widely produced and used as substitute compounds. ${ }^{10}$ Previous studies have reported that perfluorobutane sulfonate (PFBS) is the most abundant perfluoroalkyl substance (PFAS) in the lower Rhine River watershed, even in drinking water, and an industrial point source was shown to be responsible. ${ }^{11-14}$ Additionally, Ahrens et al. $^{15}$ found that perfluorobutanoic acid (PFBA) is the dominant perfluoroalkyl carboxylate (PFCA) in the air of landfill sites, and Cai et al. ${ }^{16}$ reported that PFBA is the dominant PFAA in the Arctic Ocean. However, few studies have investigated the distribution and transport of short-chain PFAAs in the environment.

Since the 1960s, Hubei Province has become one of the production bases of the fluorochemical industry in China. In the early days, the fluorochemical industry focused on the production of hydrofluoric acid and fluoro fine chemicals. In the late 1990s, the organic fluorochemical industry got a rapid development because of its great market. Recently, due to the phase-out of PFOS-based products and the restriction of PFOS in industrial and consumer products by international organizations, the development and production of substitute compounds of eight-carbon homologue products has been increasing in Hubei. Wang et al. found high levels of PFAAs

Received: $\quad$ May 12, 2013

Revised: July 24, 2013

Accepted: July 24, 2013

Published: July 24, 2013 
from a manufacturing plant and its ambient environment in Wuhan, Hubei Province, with a highest value in dust of 4962 $\mu \mathrm{g} / \mathrm{g} \mathrm{dw}$ for PFOS and $160 \mu \mathrm{g} / \mathrm{g} d \mathrm{w}$ for PFOA. ${ }^{17}$ Tangxun Lake is located in Wuhan, covering a total area of $32 \mathrm{~km}^{2}$. There is a new and high-tech industrial development zone that stretches from the north to the east of the lake, including industries such as electronics, pharmaceuticals, new materials, and chemical industries. In the upstream part of the lake there is a wastewater treatment plant (WWTP) that primarily serves the new and high-tech industrial development zone. The daily sewage treatment capacity of the WWTP is 50000 tons of wastewater, and its effluent is directly discharged into the lake through a drainage channel. In addition to natural rainfall, wastewater is the main source of water in Tangxun Lake. In our preliminary experiments, we found that there were high levels of short-chain PFAAs in the effluent of the WWTP (Table S4 of the Supporting Information). It caused us to consider what the impacts are and how PFAAs, especially short-chain PFAAs, distribute in the aquatic environment.

The specific objectives of this study were to evaluate the distribution of PFAAs, especially short-chain PFAAs in water and sediments in Tangxun Lake, China, and to investigate the bioaccumulation of short-chain PFAAs in aquatic biota, including plants and fish. To accomplish these goals, a total of 13 PFAAs, including 10 PFCAs (C4-C13) and three perfluoroalkane sulfonates (PFSAs, C4, C6, C8), were analyzed in surface water, sediment, and biological samples collected from Tangxun Lake. We estimated the distribution coefficients $\left(K_{\mathrm{d}}\right)$ to evaluate the sorption of short-chain PFAAs to sediments. In addition, bioconcentration factors (BCFs) were calculated based on the concentrations of PFAAs in biological and water samples. Different transport pathways and environmental behaviors between short-chain and longer chain PFAAs were also discussed in this study.

\section{MATERIALS AND METHODS}

Chemicals. Samples were analyzed for 13 PFAAs, including PFBA, perfluoropentanoic acid (PFPeA), perfluorohexanoic acid (PFHxA), perfluoroheptanoic acid (PFHpA), perfluorooctanoic acid (PFOA), perfluorononanoic acid (PFNA), perfluorodecanoic acid (PFDA), perfluoroundecanoic acid (PFUnDA), perfluorododecanoic acid (PFDoDA), perfluorotridecanoic acid (PFTrDA), PFBS, perfluorohexane sulfonate (PFHxS), and PFOS. Nonlabeled calibration standards and isotopically labeled internal standards were obtained from Wellington Laboratories (Ontario, Canada). A list with abbreviations of the standards used is shown in Table S1.

Sampling. A sampling map and sample sites are shown in Figure S1. In the upstream part of Tangxun Lake, municipal and industrial wastewater is discharged into Tangxun Lake through drainage channel $\mathrm{H} 1$, including direct discharge of untreated wastewater and WWTP effluents. Downstream, Tangxun Lake is connected with the Changjiang River through channel H2. A total of 21 sampling sites ( $M$ and S1-S20) were selected in the area of Tangxun Lake. Specifically, $M$ was located at drainage channel $\mathrm{H} 1$, while $\mathrm{S} 1-\mathrm{S} 20$ were located at specific intervals along the water flow direction of the lake at 0-2 km (S1-S5), 2-4 km (S6-S8), 4-6 km (S9-S14), 6-8 $\mathrm{km}(\mathrm{S} 15-18)$, and $8-12 \mathrm{~km}$ (S19 and S20) from the drainage outlet. Overall, surface water samples were collected from $M$ and S1-S20 using 1L polypropylene (PP) bottles that had been precleaned with methanol. For sample collection, bottles were submerged to approximately $0.4 \mathrm{~m}$ below the surface, uncapped, and filled, and then bottles were recapped underwater. Additionally, 18 sediment samples were collected at S1-S18 and stored in PP bags. Only surface sediment (about 3-5 cm deep) was collected using a grab sampler (Wildco Ekman Grab, $152 \times 152 \times 152 \mathrm{~mm}$, Buffalo, NY, USA). Moreover, two sediment cores were collected at S2 (C1) and S3 (C2). Sediment cores were collected using an acrylic tube (120 cm long and $12 \mathrm{~cm}$ i.d.), after which the cores were sliced at $5 \mathrm{~cm}$ intervals up to 45 and $40 \mathrm{~cm}$ for $\mathrm{C} 1$ and $\mathrm{C} 2$, respectively, and then stored in PP bags. Additionally, biological samples were collected at S2 and stored in PP bags. Biological samples included plants (lotus root (Nelumbo nucifera; $n=3$ ), common duckweed (Lemna minor; $n=3$ ), common water hyacinth (Eichhornia crassipes; $n=3$ ), and fish (crucian carp (Carassius carassius; $n=8$ ) and the sharpbelly (Hemiculter leucisculus; $n=7)$ ). Plants were washed and homogenized as one pooled sample for each species. Muscle tissues were obtained from fish samples. Surface water samples were collected in April, September, and October 2011; sediment and biological samples were collected in October 2011. All samples were stored in an ice-box after collection and transported to the laboratory. Water samples were kept at 4 ${ }^{\circ} \mathrm{C}$, while other samples were frozen and kept at $-20{ }^{\circ} \mathrm{C}$ until further analysis.

Sample Pretreatment. Surface water samples were pretreated as previously described by Taniyasu et al. ${ }^{18}$ Briefly, $500 \mathrm{~mL}$ of sample was prefiltered through a $0.45 \mu \mathrm{m}$ glass fiber filter membrane and then spiked with 2 ng of ${ }^{13} \mathrm{C}_{4}-\mathrm{PFOS}$, ${ }^{13} \mathrm{C}_{4}-$ PFOA, ${ }^{13} \mathrm{C}_{4}-$ PFBA, and ${ }^{13} \mathrm{C}_{2}-$ PFDoDA as internal standards. The samples were subsequently loaded onto an Oasis WAX SPE cartridge that had been preconditioned with 4 $\mathrm{mL}$ of $0.1 \%$ ammonium hydroxide (in methanol), $4 \mathrm{~mL}$ of methanol, and $4 \mathrm{~mL}$ of Millipore water at a rate of one drop/s. Cartridges were washed with $4 \mathrm{~mL}$ of buffer $(25 \mathrm{mM}$ acetic acid/ammonium acetate, $\mathrm{pH} 4$ ) and then centrifuged for 10 $\mathrm{min}$ at $3000 \mathrm{rpm}$ to remove the residual water. Target compounds were eluted with $4 \mathrm{~mL}$ of methanol and $4 \mathrm{~mL}$ of $0.1 \%$ ammonium hydroxide (in methanol). Finally, the eluate was concentrated to $1 \mathrm{~mL}$ under nitrogen for injection.

Sediment and biological samples were air-dried and homogenized. Sediment samples were sieved through a stainless steel 50 -mesh $(0.3 \mathrm{~mm})$ sieve, after which they were extracted using a methanol-based extraction method ${ }^{4}$ with minor modifications. Briefly, $0.1 \mathrm{~g}$ of sediment (dry sample) with $2 \mathrm{ng}$ internal standards was sonicated in $10 \mathrm{~mL}$ of methanol at $60^{\circ} \mathrm{C}$ for $30 \mathrm{~min}$. After shaking $(250 \mathrm{rpm})$ for $16 \mathrm{~h}$ and centrifugation, the supernatant was concentrated to $1-2$ $\mathrm{mL}$ under nitrogen and then diluted with $50 \mathrm{~mL}$ of water. The dilution was then loaded onto a preconditioned Oasis WAX cartridge. The SPE procedures were the same as those for the water samples. Biological samples were extracted using an alkaline digestion method. ${ }^{18}$ Briefly, $0.2 \mathrm{~g}$ of a freeze-dried biological sample with $2 \mathrm{ng}$ of internal standards was sonicated in $10 \mathrm{~mL}$ of $10 \mathrm{mM} \mathrm{KOH}$ (in methanol) at $60{ }^{\circ} \mathrm{C}$ for $30 \mathrm{~min}$. The following procedures were the same as those for the sediment samples. The total organic carbon (TOC) of surface sediment and sediment core samples was measured using an O.I. Analytical Solids TOC Analyzer (O.I. Analytical, USA).

Instrument Analysis and QA/QC. PFAAs analysis was accomplished using a high performance liquid chromatograph (Ultimate 3000 HPLC, Dionex, Sunnyvale, CA, USA) equipped with an electrospray ionization tandem mass spectrometer (API 3200, Applied Biosystems/MDS SCIEX, 

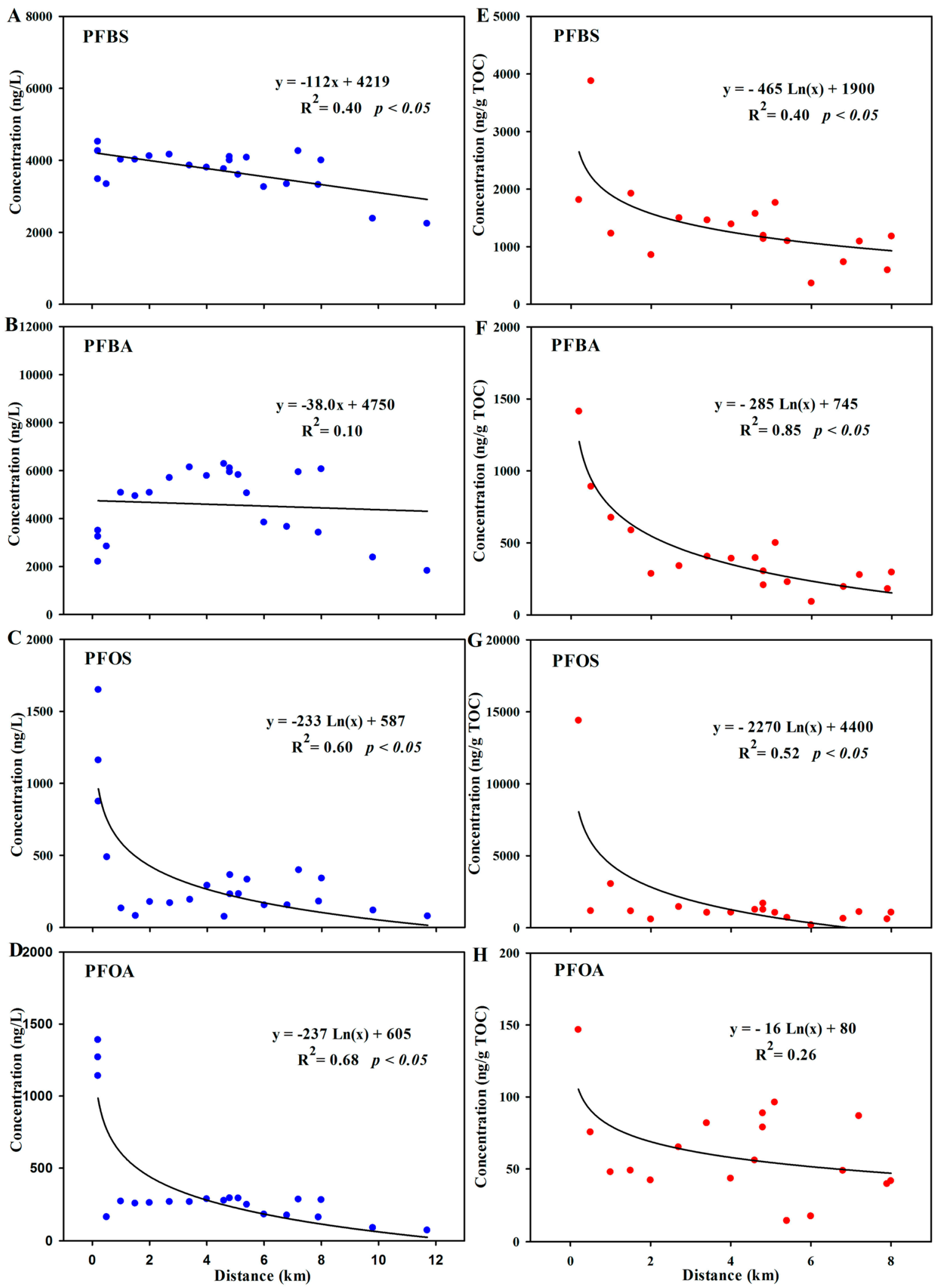

Figure 1. Spatial distribution of PFBS, PFBA, PFOS, and PFOA in surface water (A-D) and sediments (E-H, TOC normalized sediment concentrations are shown).

US) operated in negative mode. The details of analyte separation were shown in the Supporting Information. Quantitative analyses were then conducted using ESI/MS/ MS. Detailed instrument parameters are shown in Table S2.
Procedural blanks were conducted for each batch of 10 samples to check for possible laboratory contamination and interferences. The procedural blank concentrations for water, sediment, and aquatic biota are shown in Table S3. Sample 
concentrations were quantified based on an internal standard calibration curve. The method limit of quantification (MLQ) was considered to be the lowest concentration resulting in a signal-to-noise ratio $(\mathrm{S} / \mathrm{N}) \geq 10$ for each compound. The mean matrix spike recovery $(n=5)$ of the PFAAs ranged from 91 to $117 \%, 95$ to $117 \%$, and 79 to $109 \%$ for water, sediment, and aquatic biota, respectively (Table S2). Duplicate samples and calibration check standards showed excellent reproducibility with a mean standard deviation of $\sim 10 \%$ for the PFAAs. Information about the quality assurance and quality control is detailed in the SI.

Data Analysis. The distribution coefficient, $K_{\mathrm{d}}$, is often used to assess the ability of chemical substances adsorbed by the solid phase (sediment, sludge) from the liquid phase (water). In the present study, the $\mathrm{K}_{\mathrm{d}}$ of all analyzed PFAAs was estimated using the following equation:

$$
K_{\mathrm{d}}=C_{\text {sediment }} / C_{\text {water }} \times 10^{3}
$$

where $C_{\text {sediment }}$ is the concentration of PFAA in sediment $(\mathrm{ng} / \mathrm{g}$ $\mathrm{dw}), C_{\text {water }}$ is the concentration of PFAA in water $(\mathrm{ng} / \mathrm{L})$, and $K_{\mathrm{d}}$ is in $\mathrm{L} / \mathrm{kg}$. Previous studies have shown that the sorption of PFAAs in sediments can be greatly affected by the organic carbon fraction $\left(f_{\mathrm{OC}}\right){ }^{19}$ Therefore, the organic carbon normalized adsorption coefficient $\left(K_{\mathrm{OC}}\right)$ was calculated using the following formula: ${ }^{20}$

$$
K_{\mathrm{OC}}=K_{\mathrm{d}} \times 100 / f_{\mathrm{OC}}
$$

Spearman rank correlation analysis and curvilinear regression were performed with IBM PASW Statistics 18.0 (SPSS Inc., 1993-2007).

\section{RESULTS AND DISCUSSION}

Surface Water. C4-C10 PFCAs and C4, C6, and C8 PFSAs were detected in all surface water samples, whereas PFUnDA, PFDoDA, and PFTrDA were below the detection limits. Upstream of Tangxun Lake (at site M), comparatively high levels of PFAAs were observed in surface water [total PFAAs (all PFAAs measured): $70400 \mathrm{ng} / \mathrm{L}$ ], with the dominant PFAAs being PFBS (15300 ng/L) and PFBA (47 800 ng/L; Table S5). The concentrations of PFOS and PFOA in water at $\mathrm{M}$ were $2130 \mathrm{ng} / \mathrm{L}$ and $2630 \mathrm{ng} / \mathrm{L}$, respectively. In WWTP effluents, the mean concentrations of PFOS and PFOA were $1630 \mathrm{ng} / \mathrm{L}$ and $2835 \mathrm{ng} / \mathrm{L}$, respectively (Table S4), comparable to those at site $\mathrm{M}$. However, the mean PFBS and PFBA levels in WWTP effluents were $4950 \mathrm{ng} / \mathrm{L}$ and $6000 \mathrm{ng} /$ L, respectively (Table S4), 5-8 times lower than those at site $\mathrm{M}$. There might be complex sources of PFAA contamination in Tangxun Lake, including untreated industrial wastewater and WWTP effluents. Furthermore, it seems that direct discharge of untreated industrial wastewater through the upstream channel was mainly responsible for the high levels of PFBS and PFBA contamination in the lake, while both untreated wastewater and WWTP effluents were the contamination source of PFOA and PFOS.

In Tangxun Lake (S1-S20), the concentrations of total PFAAs in the surface water ranged from 4570 to $11890 \mathrm{ng} / \mathrm{L}$ (Table S5), which were lower than those upstream (M). The predominant PFAAs were PFBS (2240-4520 ng/L) and PFBA (1820-6280 ng/L), which accounted for $32-49 \%$ and $22-$ $56 \%$ of the total PFAAs (calculated based on the weight basis), respectively (Figure S2). In a previous study, PFBS and PFBA were also the most abundant components in water samples collected from the Rhine River watershed around industrial point sources, but their concentrations (up to $181 \mathrm{ng} / \mathrm{L}$ and $335 \mathrm{ng} / \mathrm{L}$, respectively) were much lower than those observed in the present study. ${ }^{14}$ Additionally, the levels of PFBS in the present study were 1 order of magnitude greater than those found in the river water around a fluorochemical industrial park specializing in the manufacture of PFBS and polytetrafluoroethylene (PTFE). ${ }^{21}$ The concentrations of PFOS and PFOA in water at sampling sites from $S 1$ to $S 20$ were in a range of 73.4$1650 \mathrm{ng} / \mathrm{L}$ (mean value, $357 \mathrm{ng} / \mathrm{L}$ ) and $70.5-1390 \mathrm{ng} / \mathrm{L}$ (mean value, 372), respectively, contributing $0.70-14 \%$ and $1.5-13 \%$ of the total PFAAs, respectively. The levels of PFOS were lower, whereas the levels of PFOA were higher than those in river water contaminated by the semiconductor and electronics industries in Taiwan. ${ }^{22}$ We also detected high levels of PFPeA, PFHxA, PFHpA, and PFHxS in water at these sites, with mean values of $74.5(26.4-254) \mathrm{ng} / \mathrm{L}, 120(27.8-462)$ $\mathrm{ng} / \mathrm{L}, 127$ (23.8-478) ng/L, and 488 (286-578) ng/L, respectively, which were much higher than those in water samples from WWTPs and lakes in previous studies. ${ }^{5,23}$ In Labadie's study, adsorption in the filtration process of water samples is observed both in nylon and glass fiber filters. Using glass fiber filters, the sorption ranges from $10 \%$ to $30 \%$ for longchain PFCAs $\left(\mathrm{CF}_{2}>10\right)$, while the sorption of PFOS and PFBS is lower than $6 \% .{ }^{24}$ In our study, PFUnDA, PFDoDA, and PFTrDA were all not observed in the surface water. It could be explained, at least in part, by the sorption to the filter.

The levels of PFAAs in water from the drainage channel $\mathrm{H} 1$ (at site M) upstream of Tangxun Lake were much higher than those in the lake (at sites S1-S20). This finding further suggested that the PFAA contamination of Tangxun Lake was mainly a result of upstream drainage. Figure 1 shows the spatial distribution of PFBS, PFBA, PFOS, and PFOA in water from the lake $(S 1-S 20)$. The concentrations of these four PFAAs in water samples gradually decreased from the drainage outlet to distant sites. However, the decreasing rates of the compound levels along the current were different between PFOS and PFOA and between PFBS and PFBA. Concentrations of PFOS and PFOA in water exponentially dropped along the water flow, whereas concentrations of PFBS and PFBA linearly declined. Within $2 \mathrm{~km}$ away from the drainage channel $\mathrm{H} 1$, levels of PFOS and PFOA in water decreased by $85 \%$ and $81 \%$ compared with those at $S 1$, respectively, while levels of PFBS and PFBA barely changed. At site S20, $11.7 \mathrm{~km}$ downstream from drainage channel $\mathrm{H} 1$, concentrations of PFOS and PFOA were $7 \%$ and $5 \%$ of those at site $S 1$, respectively. However, at the same site (S20), the levels of PFBS and PFBA were still $64 \%$ and $54 \%$ of those at $S 1$, respectively. This is an interesting finding and has implications for the worldwide distribution of PFAAs in aquatic environments.

Surface Sediments. PFBS, PFBA, PFOS, PFOA, and PFHxS were detected in all surface sediment samples collected from Tangxun Lake, whereas PFPeA, PFHxA, and PFNA were not detected. The concentrations of total PFAAs in the surface sediments from Tangxun Lake ranged from $41.8 \mathrm{ng} / \mathrm{g} \mathrm{dw}$ to $800 \mathrm{ng} / \mathrm{g} \mathrm{dw}$ (Table S5). PFBS and PFOS were the most abundant PFAAs in sediments with levels ranging from 21.1 to $114 \mathrm{ng} / \mathrm{g} \mathrm{dw}$ and 10.9 to $623 \mathrm{ng} / \mathrm{g} \mathrm{dw}$, accounting for $42 \%$ and $38 \%$ of the total PFAAs on average, respectively (Figure S2). PFBA was the next most abundant PFAA with a mean concentration of $16.3(5.26-61.2) \mathrm{ng} / \mathrm{g} \mathrm{dw}$, accounting for an average of $12 \%$ of the total PFAAs. The concentrations of PFOS in sediments collected from Tangxun Lake were 2-3 
orders of magnitude higher than those observed in the Baiyangdian Lake and Taihu Lake in China and comparable with those in Lake Ontario. ${ }^{25-27}$ Additionally, the levels of PFBS observed in the present study were 1 or 2 orders of magnitude greater than those in sediments around a fluorochemical industrial park specializing in the manufacture of PFBS and PTFE. ${ }^{21}$ The mean levels of PFOA and PFHxS in surface sediments were $2.35 \mathrm{ng} / \mathrm{g} \mathrm{dw}$ and $3.49 \mathrm{ng} / \mathrm{g} \mathrm{dw}$, respectively. Since the main sorption interactions of the PFAAs are hydrophobic in nature, the concentration in the sediment should correlate with the organic carbon fraction $\left(f_{\mathrm{OC}}\right){ }^{19}$ Figure 1 shows the spatial distribution of four dominant PFAAs in the surface sediments of Tangxun Lake. The TOC normalized concentrations of PFBS, PFBA, PFOS, and PFOA in sediments decreased logarithmically from the drainage outlets to distant sites.

The average $K_{\mathrm{d}}$ values for sediments collected from Tangxun Lake ranged from $3.69 \mathrm{~L} / \mathrm{kg}$ (PFBA) to $19800 \mathrm{~L} / \mathrm{kg}$ (PFUnDA). The log $K_{\mathrm{d}}$ of PFAAs in Tangxun Lake was in the same range as those determined in Tokyo Bay of Japan and in aquatic systems of The Netherlands. ${ }^{28,29}$ The log $K_{\mathrm{OC}}$ ranges were $1.7-5.6$ for the $\mathrm{C} 4-\mathrm{C} 12$ carboxylic acids and 2.2-3.7 for the $\mathrm{C} 4, \mathrm{C} 6$, and $\mathrm{C} 8$ sulfonates. In this study, the $\log K_{\mathrm{OC}}$ values of PFOS $(3.7 \pm 0.38)$ and PFOA $(2.3 \pm 0.23)$ were consistent with the results in laboratory partitioning experiments reported by Ahrens et al (PFOS, $3.7 \pm 0.56$; PFOA, $2.4 \pm 0.12$ ). ${ }^{30}$ Interestingly, a positive correlation was observed between the average $\log K_{\mathrm{OC}}$ and the perfluoroalkyl chain length for C8C12 PFCAs $(p<0.05)$, whereas the $\log K_{\mathrm{OC}}$ values were nearly invariable with a mean range of 1.8-2.3 for short-chain PFCAs and lower for C13 PFCAs than C12 PFCAs (Figure 2A). Meanwhile, with the same perfluoroalkyl chain length, the average $\log K_{\mathrm{OC}}$ values for PFSAs were $0.2 \log$ units higher than those for PFCAs, such as PFBS (2.5) vs PFPeA (2.3), PFHxS (2.1) vs PFHpA (1.9), and PFOS (3.7) vs PFNA (3.5).

Different $\log K_{\mathrm{OC}}$ values reflected different distributions and sorption potentials of PFAAs in water-sediment environment. Since hydrophobic interactions are considered important in the distribution and sorption of PFAAs in the environment, a correlation with $\log K_{\mathrm{OW}}$ would be expected. In the study by Arp et al., they carefully validated the consistency and reliability of the $K_{\mathrm{OW}}$ values for PFAAs predicted from four types of software packages, including EPI Suite, ClogP, SPARC, and COSMOtherm. ${ }^{31}$ On the basis of their results, they believed that EPI Suite and ClogP are not suitable for PFAAs. ${ }^{31}$ In 2009, experimentally determined partition coefficients for PFAAs were obtained using a voltammetric method. ${ }^{32}$ In 2011, Wang et al. presented new results of $\log K_{\text {OW }}$ using the COSMOtherm model. ${ }^{33}$ The $\log K_{\mathrm{OW}}$ values predicted from COSMOtherm in Wang et al.'s study were about one log unit higher than those predicted from COSMOtherm in Arp et al.'s study, but almost $3.5 \mathrm{log}$ units higher than those obtained from experimental determination (Table S9). Although different log $K_{\mathrm{OW}}$ values were predicted by the different methods from the three literature sources, a significantly positive correlation was observed between all these values and the perfluoroalkyl chain length of PFCAs (Figure S3). Considering the availability for C4-C13 PFCAs and C4, C6, and C8 PFSAs, we discussed the relation between $K_{\mathrm{OW}}$ and $K_{\mathrm{OC}}$ using the values calculated from COSMOtherm method in Wang's report. For C8-C12 PFCAs and C6 and C8 PFSAs, a significantly positive correlation was found between the average $\log K_{\mathrm{OC}}$ and the $\log K_{\mathrm{OW}}(p<0.05$; Figure $2 \mathrm{~B}$ ). The above-mentioned results indicated that the
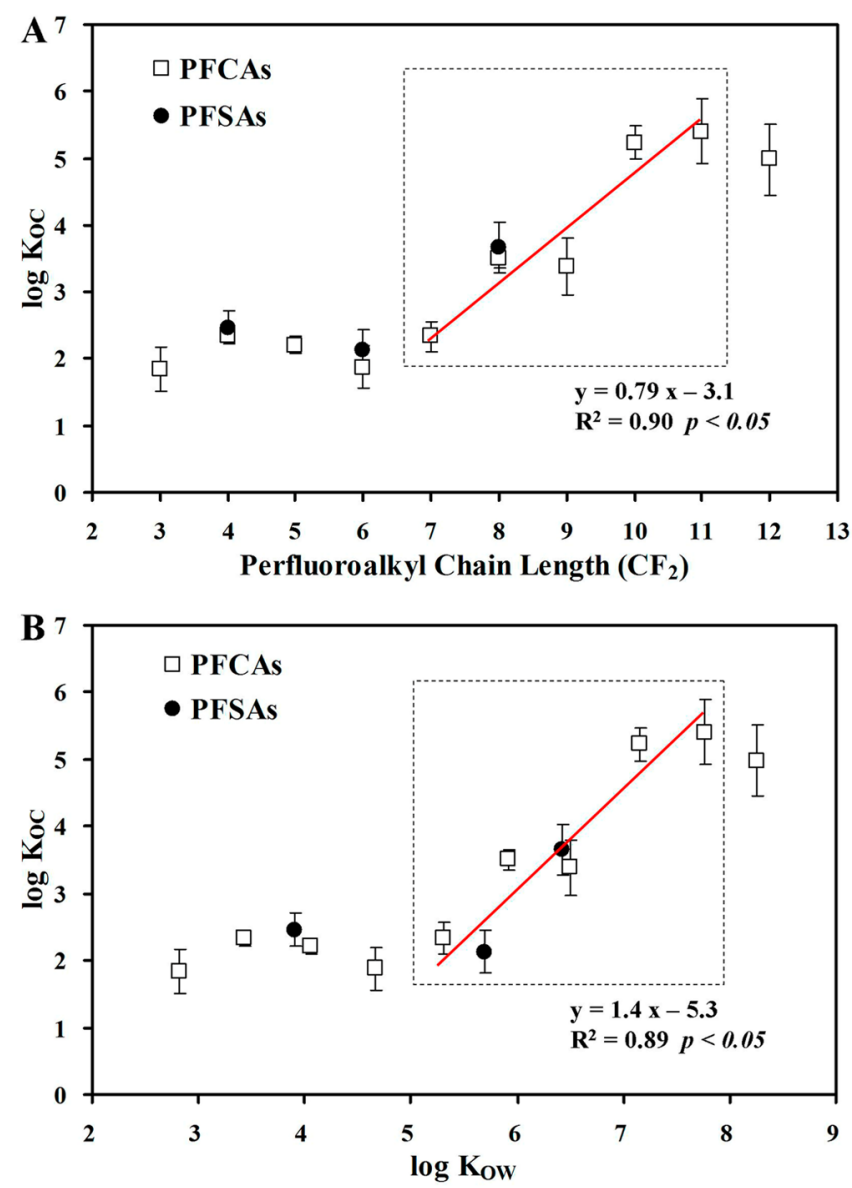

Figure 2. The relationship between the average log $K_{\mathrm{OC}}$ and the perfluoroalkyl chain length $\left(\mathrm{CF}_{2}\right)(\mathrm{A})$ and between the $\log K_{\mathrm{OC}}$ and $\log K_{\mathrm{OW}}(\mathrm{B})$.

sediment-water distribution of C8-C12 PFCAs and C6 and C8 PFSAs was mainly affected by hydrophobic interactions, and the sediment sorption potential was increased with the increasing perfluoroalkyl chain length. It was consistent with the previous studies that the perfluorocarbon chain length is the dominant structural feature influencing the sorption. ${ }^{19,28}$ Therefore, compounds with higher $K_{\mathrm{OW}}$ and $K_{\mathrm{OC}}$ values are more frequently encountered in sludge than those with lower values. ${ }^{34}$ However, in the present study, the values of $\log K_{\mathrm{OC}}$ for short-chain PFCAs with lower log $K_{\mathrm{OW}}$ values were nearly invariable. We hypothesized that short-chain PFCAs were more hydrophilic. In contrast to those of PFCAs with longer chains, the sediment-water distributions of short-chain PFCAs were not mainly affected by hydrophobic interactions and perfluoroalkyl chain length. Consistent with the results of $\log K_{\mathrm{OC}}$, with the same perfluoroalkyl chain length, the $\log K_{\mathrm{OW}}$ values for PFSAs were almost $0.5 \mathrm{log}$ units (calculated values ${ }^{33}$ ) or $0.2 \mathrm{log}$ units (experimental values ${ }^{32}$ ) higher than those for PFCAs. This might be due to the small size of the carboxylate moiety, leading to being slightly more hydrophilic as compared with the sulfonate moiety. ${ }^{19}$

Considering partitioning behavior alone, PFBS and PFBA are less likely to be in sediment than PFOS and PFOA, which, for the most part, can be accounted for by simple decreasing molar volume attributed to the $\mathrm{CF}_{2}$ moiety. These findings are considered to explain the different spatial distributions in surface water between PFBS, PFBA, PFOS, and PFOA. Because 

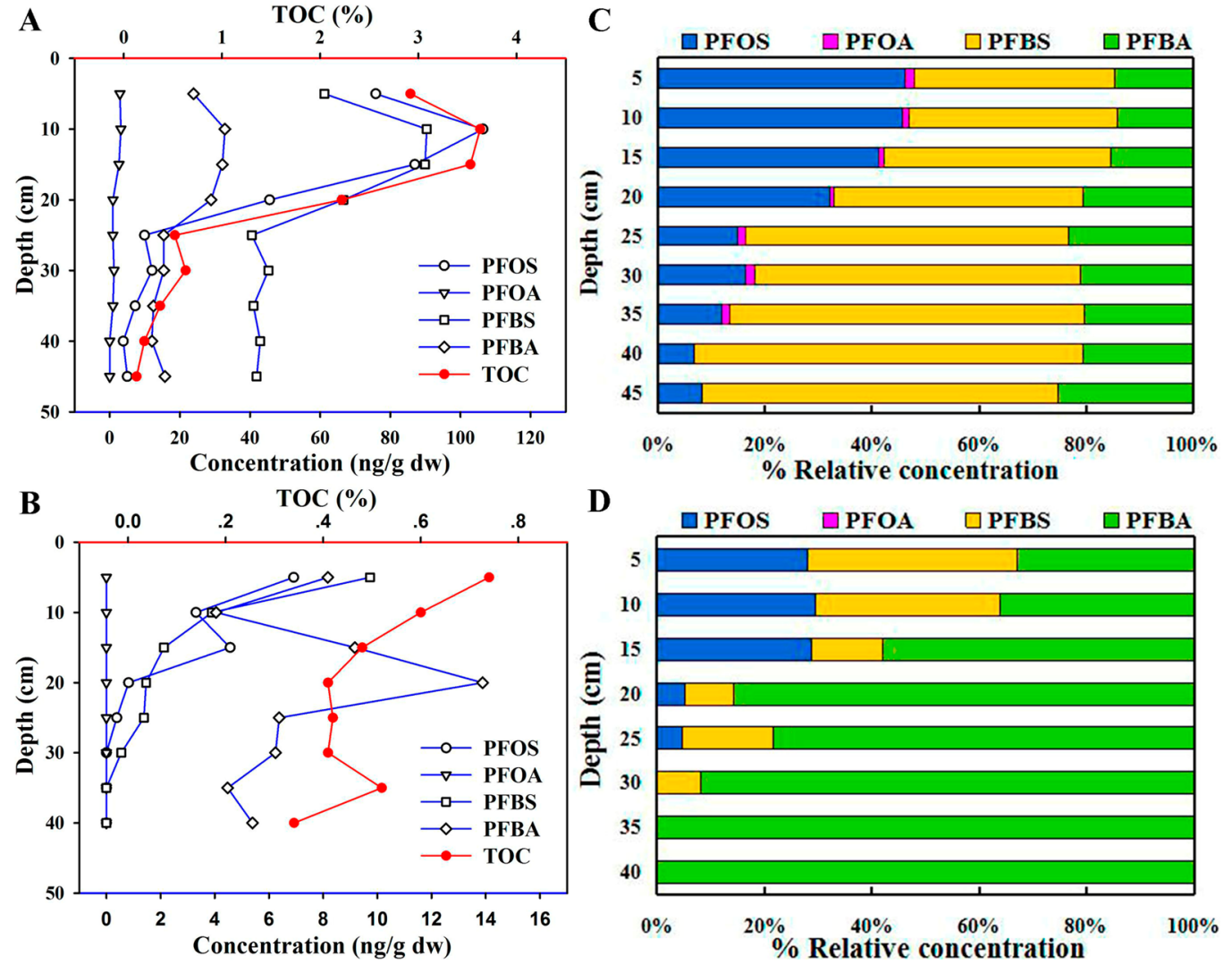

Figure 3. Vertical concentration profiles and relative composition of PFBS, PFBA, PFOS, and PFOA in sediment core C1 (A and C) and sediment core $\mathrm{C} 2$ (B and D).

they have a better water solubility than PFOS and PFOA, PFBS and PFBA could be dissolved in water and be transported along the water current. However, PFOS and PFOA could be adsorbed by sediments during water flow, resulting in logarithmically decreased concentrations of these compounds as water travels downstream.

Sediment Cores. Two sediment cores from Tangxun Lake were analyzed for PFAAs to assess the vertical transport trends. The concentrations of total PFAAs in two sediment cores ranged from 58.8 to $238 \mathrm{ng} / \mathrm{g} \mathrm{dw}$ (C1) and 5.07 to $28.2 \mathrm{ng} / \mathrm{g}$ $\mathrm{dw}$ (C2), respectively (Table S8). PFBS, PFOS, and PFBA were the dominant PFAAs in both sediment cores, and the sum levels of these three contaminants accounted for more than $90 \%$ of the total PFAAs. Because of the phase-out of PFOSbased products and the restriction of PFOS in industrial and consumer products by international organizations, short-chain PFAAs have been implemented as substitute compounds for eight-carbon products. ${ }^{35}$ A previous study focusing on the PFAA deposition in peat cores reported the production shift from long-chain to short-chain PFAAs. ${ }^{36}$ Meanwhile, our results corroborated with the premise that short-chain homologues are emerging in China as replacements for eightcarbon chain PFAAs.

The TOC ranged from $3.6 \%$ to $0.13 \%$ for $\mathrm{C} 1$ and $0.74 \%$ to $0.34 \%$ for $\mathrm{C} 2$. A positive correlation was observed between the TOC and levels of PFBS, PFBA, PFOS, and PFOA in C1, whereas no obvious relationships were found between TOC and levels of these contaminants except for PFBS in C2 $(p<$ 0.05 ). Figure 3 shows the vertical distributions of PFAAs and TOC in two sediment cores. The concentrations of PFAAs and TOC fractions decreased with increasing depth in the sediment cores. TOC fractions in $\mathrm{C} 1$ in the upper layers $(5-20 \mathrm{~cm})$ were 1 order of magnitude greater than those in the deeper sediment layers $(25-45 \mathrm{~cm})$, whereas only a slight decline with the increase of depth for $\mathrm{C} 2$ was detected. Considering the geochemical characteristics of $\mathrm{C} 2$, for which no obvious relationships were observed between TOC and PFOS or PFBA levels, we assumed that the vertical distributions of PFAAs in C2 could be useful for simplistic assessment of the transport trends of contaminants in a homogeneous phase. As depth increased, the proportion of PFBA in C2 gradually increased, while that of PFOS gradually decreased (Figure 3). According to the distribution coefficient, PFOS may be strongly absorbed to sediment. However, PFBA has a lower log $K_{\mathrm{OC}}$, resulting in a better water solubility but a weaker sediment sorption potential. In wet sediment, PFBA might be mainly dissolved in pore water, whereas PFOS might be primarily adsorbed by the sediment solid phase. A study that investigated the partitioning behavior of PFAAs between pore water and sediment in sediment cores revealed that short-chain PFCAs are present exclusively in pore water, while long-chain PFCAs are only found in sediment solid phase. ${ }^{29}$ In that work, the concentrations of PFAAs found in air-dried sediment samples are several orders of magnitude higher than those found in the sediment solid, and it could be explained considering the effect of pore water. ${ }^{29}$ In dried sediment samples, PFAAs dissolved originally in the pore water in wet sediment samples could remain in the dry sediment solid. Investigators have reported that adsorption or limited penetration of the anionic PFAAs into the sediment organic matter is the dominant mechanism of sorption. ${ }^{19}$ The sediment concentrations of PFOS and longchain PFCAs in the deeper layers might be increased by 
deposition and not be due to a downward migration in pore water. ${ }^{29}$ Dreyer et al. ${ }^{36}$ also described such different vertical mobility for PFAAs of different perfluoroalkyl chain lengths. Consistent with our results, they showed that long-chain PFCAs are predominantly observed in the upper part, whereas concentration curves of shorter chain PFCAs and PFSAs were rather flat and characterized by broad peaks. ${ }^{36}$ As water percolates downward in the vertical direction of sediment core C2, PFOS would be gradually adsorbed by the sediment solid phase, while PFBA would infiltrate deeper, eventually dissolving in the pore water.

However, in sediment $\mathrm{C} 1$, there was a positive correlation between the TOC and levels of PFBS, PFOS, and PFBA. C1 is a complex environmental matrix with great variation in TOC from the upper to deeper layers. The variations of proportions of PFOS and PFBA in $\mathrm{C} 1$ were consistent with those for $\mathrm{C} 2$, but those of PFBS differed between these two sediment cores (Figure 3). An obvious correlation was observed between TOC and the levels of PFBS in both cores. The variations in TOC with depth could be explained by the differences in transport trends for PFBS in sediment cores.

Bioaccumulation. Several studies have reported the bioaccumulation or bioconcentration of PFAAs in aquatic species. ${ }^{37-39}$ However, most reports focused on PFAAs with seven perfluoroalkyl carbons or more, especially PFOS. Few experimental results in the bioaccumulation or bioconcentration of short-chain PFAAs in aquatic species have been published because of the low detection rate in the previous research. Biological samples were collected from Tangxun Lake, including two types of freshwater fish and three types of aquatic plants (at site S2). In contrast to the water samples, PFOS was the predominant PFAA in the muscle of fish, accounting for 94\% and $93 \%$ of the total PFAAs for crucian carp (mean value of $263 \mathrm{ng} / \mathrm{g} \mathrm{ww}$ ) and the sharpbelly (mean value of $348 \mathrm{ng} / \mathrm{g}$ $w w)$, respectively (Table S10). The concentration of PFOS in muscle of fish from Tangxun Lake was higher than that in fish from Lake Möhne and the Orge River, ${ }^{40,41}$ and it was comparable to the levels observed after an accidental release at Toronto Airport and those in harbor seals from the Dutch Wadden Sea. ${ }^{42,43}$ Contrary to those in water, long-chain PFCAs, such as PFUnDA, PFDoDA, and PFTrDA, were detected in all fish samples. The mean levels of PFUnDA, PFDoDA, and PFTrDA (long-chain PFCAs) in crucian carp were $2.62,6.02$, and $1.94 \mathrm{ng} / \mathrm{g}$ ww, respectively, while those in the sharpbelly were $6.94,12.5$, and $3.93 \mathrm{ng} / \mathrm{g} \mathrm{ww}$, respectively. Although relatively high levels of PFBS $(3340 \mathrm{ng} / \mathrm{L})$ and PFBA $(2840 \mathrm{ng} / \mathrm{L})$ were detected in water at site S2, the concentrations of these two compounds in the muscle of fish at this site were relatively low, ranging from $<$ MLQ to $3.61 \mathrm{ng} /$ g ww. Meanwhile, the other short-chain PFAAs, such as PFPeA, PFHxA, and PFHpA, were all below the detection limits. The average concentrations of $\mathrm{PFHxS}$ in the muscle of fish were 0.89 and $0.15 \mathrm{ng} / \mathrm{g}$ ww for crucian carp and the sharpbelly, respectively.

Three representative types of aquatic plants were collected from the same sampling site, including lotus root, common duckweed, and common water hyacinth. The composition of PFAAs in plants was different from that in fish (Table S10). In lotus root, the mean concentrations of PFBS and PFBA were $0.50 \mathrm{ng} / \mathrm{g}$ ww and $4.66 \mathrm{ng} / \mathrm{g}$ ww, respectively, while the other analyzed PFAAs were all below the detection limits. In common duckweed and common water hyacinth, PFBS, PFOS, and PFBA were the predominant PFAAs, with mean concentrations of $10.8,33.7$, and $7.62 \mathrm{ng} / \mathrm{g}$ ww in common duckweed and 5.96, 5.14, and $11.7 \mathrm{ng} / \mathrm{g}$ ww in common water hyacinth, respectively. In common duckweed, the average levels of PFUnDA and PFDoDA were 0.24 and $0.64 \mathrm{ng} / \mathrm{g}$ ww, respectively, while PFTrDA was undetected. However, these three long-chain PFAAs were all below the detection limits in common water hyacinth. Overall, the levels of short-chain PFAAs in plants were higher than those in fish, while the levels of long-chain PFAAs in plants were much lower than those in fish.

BCFs were calculated on the basis of the concentrations of PFAAs in biological organisms with wet weight and water (at site S2). Log BCF values ranged from -0.75 to 5.1 for the 13 analyzed PFAAs in all biological organisms (Table S10). These results indicated that the values of $\log$ BCFs for long-chain PFAAs were higher than those for short-chain PFAAs in both fish and plants. Furthermore, the values of $\log B C F$ s for shortchain PFAAs, including PFBS, PFBA, PFPeA, PFHxA, PFHpA, and $\mathrm{PFHxS}$, were all relatively low $(<1)$, indicating that there may be no bioaccumulation potential. The log BCF values for PFCAs with 11-13 carbons ranged from 3.0 to 4.0 for aquatic plants and from 4.3 to 5.1 for fish, which were higher than those for other PFCAs and PFOS. These findings were in accordance with the trend observed in rainbow trout and the European chub, ${ }^{38,40}$ suggesting that bioaccumulation potentials for long-chain PFCAs $(\mathrm{C}>10)$ were higher than those for PFOS. However, the values of log BCFs for PFTrDA (C13) were slightly lower than those for PFUnDA (C11) and PFDoDA (C12). It should be noted that although the bioaccumulation potential increased with increasing carbon length, bioconcentration for PFTrDA was lower than expected. This observation could be interpreted by a viewpoint that the bioaccumulation potential of PFCAs with more than 12 fluorinated carbons might be limited by their lager molecular size. ${ }^{44}$ Since direct uptake from water is probably the major route for waterborne xenobiotics, it could be hypothesized that the gills appear to be less permeable to PFTrDA than shorter PFCAs. ${ }^{38}$ The values of log BCFs for PFOA were much lower than those for PFOS, which were consistent with the reports in the Orge River and Lake Ontario. ${ }^{39,40}$ Furthermore, the values of $\log$ BCFs for short-chain PFAAs in aquatic plants were higher than those in fish, whereas the values of log BCFs for long-chain PFAAs in aquatic plants were less than those in fish.

Environmental Implications. Because of the phase-out of PFOS-based products and the restriction of PFOS in industrial and consumer products by international organizations, shortchain PFAAs have been implemented as substitute compounds for eight-carbon products. Our results confirmed the different transport rates of PFBS, PFBA, PFOS, and PFOA in the surface water, sediment, and aquatic biota of Tangxun Lake in China. The sorption in sediment and accumulation in biota of individual PFAAs differed depending on its perfluorocarbon chain length and functional group. PFBS and PFBA tended to have lower adsorption potentials than PFOS and PFOA, and therefore remained in the water phase. PFBS and PFBA also moved farther horizontally and vertically with water diffusion and permeation. It indicated that as substitute compounds for eight-carbon products the short-chain PFAAs might be very persistent and also much more mobile than PFOA and PFOS. Although there is no potential for bioaccumulation of PFBS and PFBA in aquatic biota, more attention should be paid to their aquatic ecological risk because of their increasing discharge and farther transportation. Furthermore, relatively 
high levels of PFOS and PFOA were also detected in Tangxun Lake, and their risks to aquatic organisms and nearby residents should be investigated in future studies.

\section{ASSOCIATED CONTENT}

\section{S Supporting Information}

Additional information regarding chemicals and materials, quality assurance, and quality control; Tables S1-S10; and Figures S1-S3. This information is available free of charge via the Internet at http://pubs.acs.org/.

\section{AUTHOR INFORMATION}

\section{Corresponding Author}

*Tel.: +86 (10) 62849182. Fax: +86 (10) 62849182. E-mail: shiyali@rcees.ac.cn.

\section{Notes}

The authors declare no competing financial interest.

\section{ACKNOWLEDGMENTS}

This work was supported by the National Natural Science Foundation of China (Nos. 41023005, 20907063), State Environmental Protection Welfare Scientific Research Project (201009026), and the National Basic Research Program of China (2009CB421605).

\section{REFERENCES}

(1) Hazard Assessment of Perfluorooctane Sulfonate (PFOS) and its salts, ENV/JM/RD(2002)17/FINAL; Organisation for Economic Cooperation and Development: Paris, 2002.

(2) Qui, Y. Study on Treatment Technologies for Perfluorochemicals in Wastewater; Kyoto University: Kyoto, Japan, 2007.

(3) Jahnke, A.; Ahrens, L.; Ebinghaus, R.; Temme, C. Urban versus Remote Air Concentrations of Fluorotelomer Alcohols and Other Polyfluorinated Alkyl Substances in Germany. Environ. Sci. Technol. 2006, 41 (3), 745-752.

(4) Benskin, J. P.; Phillips, V.; St. Louis, V. L.; Martin, J. W. Source Elucidation of Perfluorinated Carboxylic Acids in Remote Alpine Lake Sediment Cores. Environ. Sci. Technol. 2011, 45 (17), 7188-7194.

(5) Boulanger, B.; Vargo, J.; Schnoor, J. L.; Hornbuckle, K. C. Detection of Perfluorooctane Surfactants in Great Lakes Water. Environ. Sci. Technol. 2004, 38 (15), 4064-4070.

(6) So, M. K.; Taniyasu, S.; Yamashita, N.; Giesy, J. P.; Zheng, J.; Fang, Z.; Im, S. H.; Lam, P. K. S. Perfluorinated Compounds in Coastal Waters of Hong Kong, South China, and Korea. Environ. Sci. Technol. 2004, 38 (15), 4056-4063.

(7) Kärrman, A.; Ericson, I.; van Bavel, B.; Darnerud, P. O.; Aune, M.; Glynn, A.; Lignell, S.; Lindström, G. Exposure of Perfluorinated Chemicals through Lactation: Levels of Matched Human Milk and Serum and a Temporal Trend, 1996-2004, in Sweden. Environ. Health Perspect. 2006, 115 (2), 226-230.

(8) The nine new POPs. An introduction to the nine chemicals added to the Stockholm Convention by the Conference of the Parties at its fourth meeting. Available online: http://chm.pops.int/ Programmes/NewPOPs/Publications/t abid/695/language/en-US/D -efault.aspx.

(9) Lim, T. C.; Wang, B.; Huang, J.; Deng, S.; Yu, G. Emission Inventory for PFOS in China: Review of Past Methodologies and Suggestions. Sci. World J. 2011, 11, 1963-1980.

(10) Directive 2006/122/EC of the European Parliament and of the Council of 12 December 2006 Amending for the 30th Time Council Directive 76/769/EEC on the Approximation of the Laws, Regulations and Administrative Provisions of the Member States Relating to Restrictions on the Marketing and Use of Certain Dangerous Substances and Preparations (Perfluorooctane Sulfonates); European Parliament and Council: Strasbourg, France, 2006.
(11) Skutlarek, D.; Exner, M.; Farber, H. Perfluorinated surfactants in surface and drinking water. Environ. Sci. Pollut. Res. 2006, 13 (5), 299307.

(12) Lange, F. T.; Wenz, M.; Schmidt, C. K.; Brauch, H. J. Occurrence of perfluoroalkyl sulfonates and carboxylates in German drinking water sources compared to other countries. Water. Sci. Technol. 2007, 56 (11), 151-158.

(13) Eschauzier, C.; Haftka, J.; Stuyfzand, P. J.; de Voogt, P. Perfluorinated Compounds in Infiltrated River Rhine Water and Infiltrated Rainwater in Coastal Dunes. Environ. Sci. Technol. 2010, 44 (19), 7450-7455.

(14) Möller, A.; Ahrens, L.; Surm, R.; Westerveld, J.; van der Wielen, F.; Ebinghaus, R.; de Voogt, P. Distribution and sources of polyfluoroalkyl substances (PFAS) in the River Rhine watershed. Environ. Pollut. 2010, 158 (10), 3243-3250.

(15) Ahrens, L.; Shoeib, M.; Harner, T.; Lee, S. C.; Guo, R.; Reiner, E. J. Wastewater Treatment Plant and Landfills as Sources of Polyfluoroalkyl Compounds to the Atmosphere. Environ. Sci. Technol. 2011, 45 (19), 8098-8105.

(16) Cai, M.; Zhao, Z.; Yin, Z.; Ahrens, L.; Huang, P.; Cai, M.; Yang, H.; He, J.; Sturm, R.; Ebinghaus, R.; Xie, Z. Occurrence of Perfluoroalkyl Compounds in Surface Waters from the North Pacific to the Arctic Ocean. Environ. Sci. Technol. 2011, 46 (2), 661-668.

(17) Wang, Y.; Fu, J.; Wang, T.; Liang, Y.; Pan, Y.; Cai, Y.; Jiang, G. Distribution of Perfluorooctane Sulfonate and Other Perfluorochemicals in the Ambient Environment around a Manufacturing Facility in China. Environ. Sci. Technol. 2010, 44 (21), 8062-8067.

(18) Taniyasu, S.; Kannan, K.; So, M. K.; Gulkowska, A.; Sinclair, E.; Okazawa, T.; Yamashita, N. Analysis of fluorotelomer alcohols, fluorotelomer acids, and short- and long-chain perfluorinated acids in water and biota. J. Chromatogr., A 2005, 1093 (1-2), 89-97.

(19) Higgins, C. P.; Luthy, R. G. Sorption of perfluorinated surfactants on sediments. Environ. Sci. Technol. 2006, 40 (23), $7251-7256$.

(20) Schwarzenbach, R. P.; Gschwend, P. M.; Imboden, D. M. Environmental Organic Chemistry; Wiley-Interscience: Hoboken, NJ 2003.

(21) Bao, J.; Liu, W.; Liu, L.; Jin, Y.; Dai, J.; Ran, X.; Zhang, Z.; Tsuda, S. Perfluorinated Compounds in the Environment and the Blood of Residents Living near Fluorochemical Plants in Fuxin, China. Environ. Sci. Technol. 2010, 45 (19), 8075-8080.

(22) Lin, A. Y.-C.; Panchangam, S. C.; Lo, C.-C. The impact of semiconductor, electronics and optoelectronic industries on downstream perfluorinated chemical contamination in Taiwanese rivers. Environ. Pollut. 2009, 157 (4), 1365-1372.

(23) Murakami, M.; Imamura, E.; Shinohara, H.; Kiri, K.; Muramatsu, Y.; Harada, A.; Takada, H. Occurrence and Sources of Perfluorinated Surfactants in Rivers in Japan. Environ. Sci. Technol. 2008, 42 (17), $6566-6572$.

(24) Labadie, P.; Chevreuil, M. Biogeochemical dynamics of perfluorinated alkyl acids and sulfonates in the River Seine (Paris, France) under contrasting hydrological conditions. Environ. Pollut. 2011, 159 (12), 3634-3639.

(25) Shi, Y.; Pan, Y.; Wang, J.; Cai, Y. Distribution of perfluorinated compounds in water, sediment, biota and floating plants in Baiyangdian Lake, China. J. Environ. Monit. 2012, 14 (2), 636-642.

(26) Yang, L.; Zhu, L.; Liu, Z. Occurrence and partition of perfluorinated compounds in water and sediment from Liao River and Taihu Lake, China. Chemosphere 2011, 83 (6), 806-814.

(27) Asher, B. J.; Wang, Y.; De Silva, A. O.; Backus, S.; Muir, D. C. G.; Wong, C. S.; Martin, J. W. Enantiospecific Perfluorooctane Sulfonate (PFOS) Analysis Reveals Evidence for the Source Contribution of PFOS-Precursors to the Lake Ontario Foodweb. Environ. Sci. Technol. 2012, 46 (14), 7653-7660.

(28) Kwadijk, C. J. A. F.; Korytar, P.; Koelmans, A. A. Distribution of Perfluorinated Compounds in Aquatic Systems in The Netherlands. Environ. Sci. Technol. 2010, 44 (10), 3746-3751.

(29) Ahrens, L.; Yamashita, N.; Yeung, L. W. Y.; Taniyasu, S.; Horii, Y.; Lam, P. K. S.; Ebinghaus, R. Partitioning Behavior of Per- and 
Polyfluoroalkyl Compounds between Pore Water and Sediment in Two Sediment Cores from Tokyo Bay, Japan. Environ. Sci. Technol. 2009, 43 (18), 6969-6975.

(30) Ahrens, L.; Yeung, L. W. Y.; Taniyasu, S.; Lam, P. K. S.; Yamashita, N. Partitioning of perfluorooctanoate (PFOA), perfluorooctane sulfonate (PFOS) and perfluorooctane sulfonamide (PFOSA) between water and sediment. Chemosphere 2011, 85 (5), 731-737.

(31) Arp, H. P. H.; Niederer, C.; Goss, K.-U. Predicting the Partitioning Behavior of Various Highly Fluorinated Compounds. Environ. Sci. Technol. 2006, 40 (23), 7298-7304.

(32) Jing, P.; Rodgers, P. J.; Amemiya, S. High Lipophilicity of Perfluoroalkyl Carboxylate and Sulfonate: Implications for Their Membrane Permeability. J. Am. Chem. Soc. 2009, 131 (6), 2290-2296.

(33) Wang, Z.; MacLeod, M.; Cousins, I. T.; Scheringer, M.; Hungerbühler, K. Using COSMOtherm to predict physicochemical properties of poly- and perfluorinated alkyl substances (PFASs). Environ. Chem. 2011, 8 (4), 389-398.

(34) Heidler, J.; Halden, R. U. Meta-Analysis of Mass Balances Examining Chemical Fate during Wastewater Treatment. Environ. Sci. Technol. 2008, 42 (17), 6324-6332.

(35) Ritter, S. K. Fluorochemicals go short. Chem. Eng. News. 2010, $88(5), 12-17$.

(36) Dreyer, A.; Thuens, S.; Kirchgeorg, T.; Radke, M. Ombrotrophic Peat Bogs Are Not Suited as Natural Archives To Investigate the Historical Atmospheric Deposition of Perfluoroalkyl Substances. Environ. Sci. Technol. 2012, 46 (14), 7512-7519.

(37) Houde, M.; Bujas, T. A. D.; Small, J.; Wells, R. S.; Fair, P. A.; Bossart, G. D.; Solomon, K. R.; Muir, D. C. G. Biomagnification of Perfluoroalkyl Compounds in the Bottlenose Dolphin (Tursiops truncatus) Food Web. Environ. Sci. Technol. 2006, 40 (13), 41384144.

(38) Martin, J. W.; Mabury, S. A.; Solomon, K. R.; Muir, D. C. G. Bioconcentration and tissue distribution of perfluorinated acids in rainbow trout (Oncorhynchus mykiss). Environ. Toxicol. Chem. 2003, 22, 196-204.

(39) Martin, J. W.; Whittle, D. M.; Muir, D. C. G.; Mabury, S. A. Perfluoroalkyl Contaminants in a Food Web from Lake Ontario. Environ. Toxicol. Chem. 2004, 38 (20), 5379-5385.

(40) Labadie, P.; Chevreuil, M. Partitioning behaviour of perfluorinated alkyl contaminants between water, sediment and fish in the Urge River (nearby Paris, France). Environ. Pollut. 2011, 159 (2), 391-397.

(41) Holzer, J.; Goen, T.; Just, P.; Reupert, R.; Rauchfuss, K.; Kraft, M.; Muller, J.; Wilhelm, M. Perfluorinated Compounds in Fish and Blood of Anglers at Lake Möhne, Sauerland Area, Germany. Environ. Sci. Technol. 2011, 45 (19), 8046-8052.

(42) Awad, E.; Zhang, X. M.; Bhavsar, S. P.; Petro, S.; Crozier, P. W.; Reiner, E. J.; Fletcher, R.; Tittemier, S. A.; Braekevelt, E. Long-Term Environmental Fate of Perfluorinated Compounds after Accidental Release at Toronto Airport. Environ. Sci. Technol. 2011, 45 (19), 8081-8089.

(43) Van de Vijver, K. I.; Hoff, P.; Das, K.; Brasseur, S.; Van Dongen, W.; Esmans, E.; Reijnders, P.; Blust, R.; De Coen, W. Tissue Distribution of Perfluorinated Chemicals in Harbor Seals (Phoca vitulina) from the Dutch Wadden Sea. Environ. Sci. Technol. 2005, 39 (18), 6978-6984.

(44) Conder, J. M.; Hoke, R. A.; Wolf, W. d.; Russell, M. H.; Buck, R. C. Are PFCAs Bioaccumulative? A Critical Review and Comparison with Regulatory Criteria and Persistent Lipophilic Compounds. Environ. Sci. Technol. 2008, 42 (4), 995-1003. 